\section{Influence of Mixing on the Azo-Coupling of 1-Naphthol and Diazotized Aniline}

\author{
José Garcia-Rosas* and Sergio Petrozzi**
}

Abstract. The azo-coupling reaction between 1-naphthol and diazotized aniline in alkaline media has been studied. It is shown that the product distribution is mixing-dependent. Reaction is rapid, and the kinetically controlled regime is reached only at high stirrer speeds.

\section{Introduction}

Generally, little attention is paid to the effect of mixing on single-phase chemical reactions. This is justified, if the reactions are sufficiently slow that mixing is accomplished, before reaction has occurred to a significant extent. Fast reactions, however, may be influenced by mixing, which then increases the time (or equivalently the reactor volume) required to achieve a certain conversion and, even more important, which may alter the product distribution relative to carrying out the reaction under chemically controlled conditions (perfect mixing). Poor mixing may lead to by-products, which have to be separated from the desired material.

Reports of mixing effects with several homogeneous, liquid-phase multiple reactions are found in the literature [1]. Failure to consider and report mixing conditions unequivocally may result in product-distribution data of fast chemical reactions which can be difficult to reproduce or, on comparing data from different sources, may be misleading. Diazo-coupling reactions between phenols and aromatic azo compounds are very common in the dyestuff industry [2]. In particular, the azocoupling reaction of 1-naphthol and diazotized aniline has been studied extensively [3-7]. However, different and to a certain extent contradictory results for the product distribution and its dependence on the
$\mathrm{pH}$ have been found [3-7]. In this work, it is shown that this azo-coupling reaction (Scheme) is mixing-dependent. Monoazo (ortho and para) and bisazo dyestuffs are produced in proportions which depend on the mixing intensity.

\section{Experimental}

\subsection{Materials}

Phenyldiazonium chloride was prepared in soln. from $\mathrm{NaNO}_{2}, \mathrm{HCl}$ and aniline (Merck, FRG). The diazotization was conducted in the classical manner [2], using a slight excess of $\mathrm{NaNO}_{2}$, which was destroyed later by adding urea. The solns. are rather unstable (thermal decomposition) [8] [9], and were, therefore, kepl in an ice-bath and used within $2 \mathrm{~h}$ of their preparation.

I-Naphthol (Merck, FRG) solns. were prepared by dissolving the solid in de-ionised water. $\mathrm{NaOH}$ was added shortly before azo-coupling.

2-(Phenylazo)-I-naphthol (I) was prepared from $\beta$ naphthoquinone (Fluka, pract.) and phenylhydrazine hydrochloride (Fluka, puriss.) as described by Zincke and Bindewald [10]. It was recrystallised twice from EtOH. M.p. $138^{\circ}$.

4-(Phenylazo)-1-naphthol (II) was prepared from diazotised aniline and 1-naphthol using the method described by Witt and Dedichen [11]. It was purified by recrystallization from $\mathrm{AcOH}$ and EtoH. M.p. $208^{\circ}$

2,4-Bis (phenylazo)-1-naphthol (III) was prepared by the method of Bamberger and Heimberg [12], i.e. from 1 -naphthol and diazotised aniline, and purified by recrystallization from aniline. M.p. $195^{\circ}$.

\subsection{Analytical Method}

The UV/VIS absorption spectra of the ortho- and para-monoazo dyestuffs and the bisazo dyestuff were measured in $0.1 \mathrm{~N} \mathrm{NaOH}, 0.1 \%(v / v)$ pyridine aq. solns.

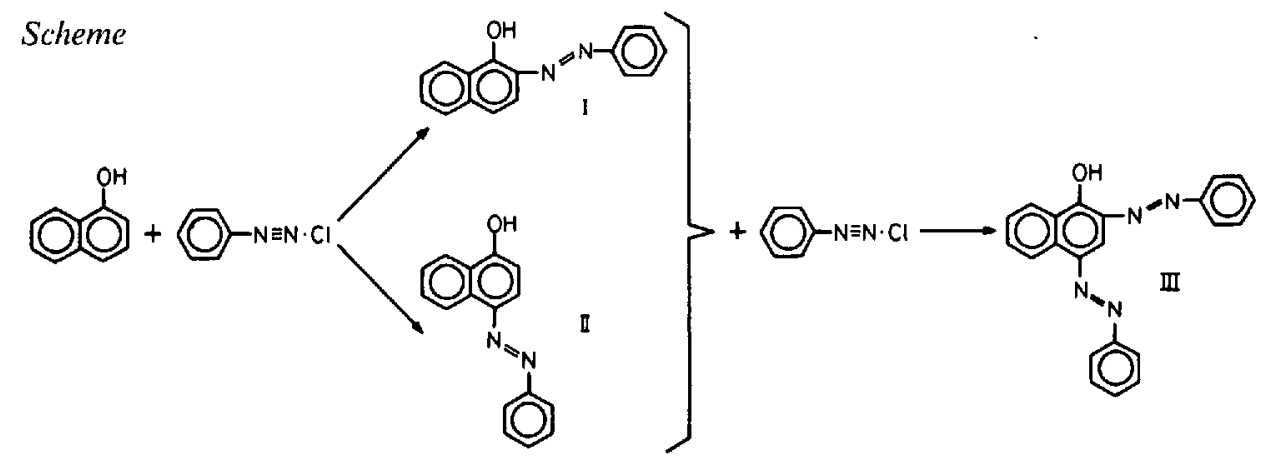

at $25^{\circ}$. The dyestuff crystals were first dissolved in pyridine and then diluted using an alkaline soln. This facilitated their dissolution. The dyestuffs particularly 2-(phenylazo)-1-naphthol (I) and 2,4-bis(phenylazo)-Inaphthol (III), have a low solubility in aq. solns. Fig. I shows the extinction coeflicients of the three dyestuffs. As reference soln. in the spectrophotometric measurements, a $0.1 \mathrm{~N} \mathrm{NaOH}, 0.1 \%$ pyridine aq. soln. was used. Absorption maxima are observed at 496 and $320 \mathrm{~nm}$ for I, 496 and $288 \mathrm{~nm}$ for II, and 540,466 , and $290 \mathrm{~nm}$ for III. Similar spectra have been obtained in $0.01 \mathrm{~N}$ $\mathrm{NaOH}, 50 \%$ pyridine aq. solns. [6].

In a mixture of the three dyes, the total absorption a is given by Eqm. I (Lamberi-Beer law), where $\varepsilon_{\mathrm{R}, 0}, \varepsilon_{\mathrm{R}, \mathrm{p}}$, and

$a=\varepsilon_{\mathrm{R}, \mathrm{o}} C_{\mathrm{R}, \mathrm{o}} l+\varepsilon_{\mathrm{R}, \mathrm{p}} C_{\mathrm{R}, \mathrm{p}} l+\varepsilon_{\mathrm{S}} C_{\mathrm{S}} l$

$\varepsilon_{\mathrm{S}}$ are the molar extinction coefficients of the orthomonoazo, para-monoazo, and bisazo derivatives, respectively. $C_{\mathrm{R} .0}, C_{\mathrm{R} . \mathrm{p}}$, and $C_{\mathrm{S}}$ are the corresponding concentrations, and $l$ is the path length. Eqm. $I$ can also be written as:

$\frac{a}{\varepsilon_{\mathrm{R}, \mathrm{o}} l}=C_{\mathrm{R}, \mathrm{o}}+\frac{\varepsilon_{\mathrm{R}, \mathrm{p}}}{\varepsilon_{\mathrm{R}, \mathrm{o}}} C_{\mathrm{R}, \mathrm{p}}+\frac{\varepsilon_{\mathrm{S}}}{\varepsilon_{\mathrm{R}, 0}} C_{\mathrm{S}}$

Eqn. 2 is of the form $t=A+B \chi+C y$. Thus, to determine the concentrations of the different species in samples of unknown composition, the absorption was measured at different wavelengths (from 270 to $570 \mathrm{~nm}$ ) and a multilinear regression analysis of Eqn. 2 was employed.

\subsection{Azo-Coupling Experiments}

Semibatch experiments were carried out, in which a soln. of diazotized aniline $\left(13 \mathrm{~mol} \cdot \mathrm{m}^{-3}, \mathrm{pH}\right.$ co. 2$), 1 / 2$ $\left(\mathrm{p} K_{1}+\mathrm{p} K_{2}\right)=11.9[2]$, was slowly added to an alkaline soln. of 1-naphthol (volume $=1.5 \times 10^{-3} \mathrm{~m}^{3}$, concentration $=1.39 \mathrm{~mol} \cdot \mathrm{m}^{-3}$ ), $\mathrm{p} K=9.8 \quad$ [2], originally present in a glass vessel. The stoichiometric ratio, defined as moles of 1 -naphthol/moles of diazotized aniline $\left(N_{\mathrm{Ao}} / N_{\mathrm{Bo}}\right)$, was 1.05-1.10. The concentrations of $\mathrm{NaOH}$ in the 1-naphthol solns. were $0.1,0.05,0.025$ 0.02 , and $0.0 \mathrm{lN}$. The corresponding measured $\mathrm{pH}$ values were as follows (given in the same order as the concentrations): before addition of the diazo component: $12.88,12.63,12.34,12.26$, and 11.92 ; after addition: $12.84,12.58,12.27,12.16$, and 11.76. A rotor-stator type mixer (Ystral $\mathrm{GmbH}$, FRG) was employed. In a previous study, the energy dissipation rate $(e)$ of the mixer has been estimated at different speeds [13]. $(\mathrm{W} / \mathrm{kg}$ ) is given for dilute aq. solns. by Eqn. 3, where $N$ is the speed in rpm:

$e=3.94 \times 10^{-10} N^{3}$

The diazotized aniline soln. was fed immediately below the mixing head in the arrangement shown schematically in Fig. 2. The experiments were performed at r.t. $\left(24 \pm 1^{\circ}\right)$. The feed rates varied between $1.7 \times 10^{-7}$ $\mathrm{m}^{3} \cdot \mathrm{s}^{-1}$ at the lowest speeds and $5 \times 10^{-7} \mathrm{~m}^{3} \cdot \mathrm{s}^{-1}$ at speeds above $5000 \mathrm{rpm}$. When the addition of diazotized aniline was completed, a sample was taken from the resulting soln. It was diluted and brought to $0.1 \mathrm{~N}$ $\mathrm{NaOH}$ and $0.1 \%$ pyridine conditions, and analysed spectrophotometrically to determine the product composition as described above. In the spectrophotometric determination, the reference soln. contained, apart from $0.1 \mathrm{~N} \mathrm{NaOH}$ and $0.1 \%$ pyridine, a quantity of I-naphthol corresponding to the slight unreacted excess over a $1: 1$ stoichiometric ratio $\left(N_{\mathrm{Ao} /} / N_{\mathrm{Bo}}\right)$. TLC confirmed the presence of the three dyestuffs in the mixture, sometimes accompanied by traces of some unidentified impurity. A mixture of $\mathrm{AcOBu} / \mathrm{NH}_{3}, 25 \%$ aq./pyridine $/ \mathrm{H}_{2} \mathrm{O}$ in the ratio $10: 10: 1: 4$ was used as eluent.

\author{
* Correspondence: Dr. J. Garcia-Rosas \\ Ciba-Geigy $A G$ \\ Klybeck \\ $\mathrm{CH}-4057$ Basel \\ ** Present address: S. Petrozzi \\ Technisch-Chemisches Laboratorium \\ ETH-Zentrum \\ CH-8092 Zürich
}




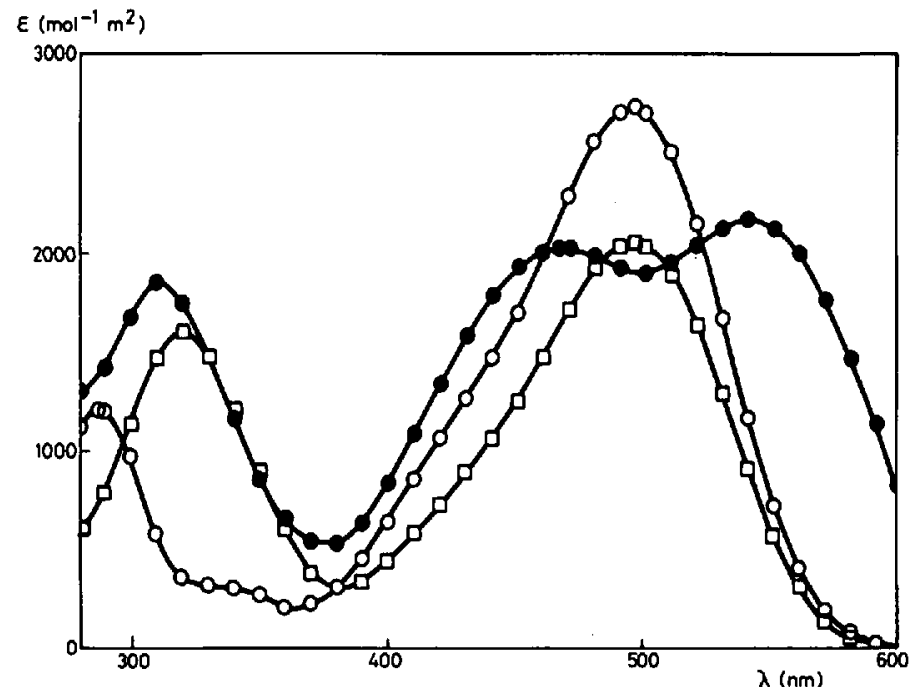

Fig. 1. Absorption spectra in $0.1 \mathrm{~N} \mathrm{NaOH}, 0.1 \%(w / v)$ pyridine aqueous solutions at $25^{\circ} . \square$ : 2-(Phenylazo)-1-naphthol (I), o: 4-(Phenylazo)-1-naphthol (II), $\bullet$ : 2,4-Bis(phenylazo)-1naphthol (III).

\section{Results and Discussion}

The azo-coupling between 1-naphthol (A) and diazotised aniline (B) can be seen as a series of parallel, consecutive reactions:
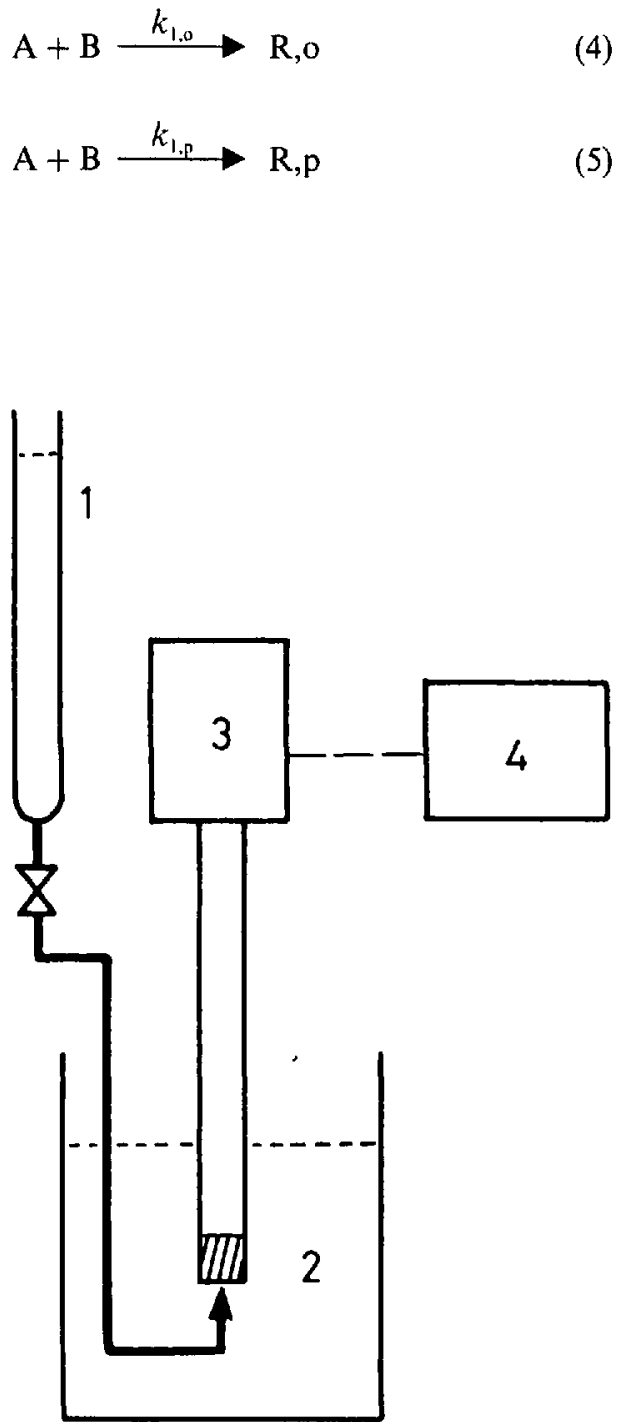

Fig. 2. Experimental set-up. 1: Diazotized aniline solution, 2 : reaction vessel, $3: \mathrm{mi}-$ xer, 4: speed governor.
$\mathrm{R}, \mathrm{O}+\mathrm{B} \stackrel{k_{2, \mathrm{p}}}{\longrightarrow} \mathrm{S}$

$\mathrm{R}, \mathrm{p}+\mathrm{B} \stackrel{k_{2,0}}{\longrightarrow} \mathrm{S}$

where $R, o$ and $R, p$ represent the orthoand para-monoazo dyestuffs, respectively, and $S$ is the bisazo dyestuff. If equimolar quantities of A and B (or, as in our case, a slight excess of A species) are allowed to react, one should expect the formation of $\mathrm{R}, \mathrm{o}, \mathrm{R}, \mathrm{p}$, and $\mathrm{S}$ species in a proportion which is solely determined by the stoichiometric ratio $\left(N_{\mathrm{Ao}} / N_{\mathrm{Bo}}\right)$ and the magnitude of the rate constants (which are $\mathrm{pH}$-dependent) for Eqns. 4-7. However, this is only true, if the reactions are carried out under chemically controlled conditions [14]. Otherwise, diffusion (and hence mixing intensity) also plays a role in determining the product distribution. The Table shows the measured product distribution as a function of the rotor speed of the mixer at different $\mathrm{NaOH}$ concentrations. At a given $\mathrm{NaOH}$ concentration, the proportion of bisazo dyestuff decreases (and thus the amount of monoazo dyestuff, ortho + para, increases) with increasing speed.
This is also represented in Fig. 3, where $X$ is defined as:

$X=2 C_{\mathrm{S}}$
$C_{\mathrm{R}, \mathrm{o}}+C_{\mathrm{R}, \mathrm{p}}+2 C_{\mathrm{S}}$

Thus, $X$ represents the fraction of limiting reagent (diazotized aniline), which has reacted to produce the bisazo compound.

At the highest $\mathrm{NaOH}$ concentration $(0.1 \mathrm{~N})$, practically only monoazo dyestuff is produced independent of the mixing intensity $(N>1000 \mathrm{rpm}$, i.e., energy dissipation rate $>0.4 \mathrm{~W} / \mathrm{kg}$ ). At lower $\mathrm{NaOH}$ concentrations, an increasing effect of the speed on the product distribution is observed (Fig. 3). Speeds lower than 1000 $\mathrm{rpm}$ and $\mathrm{NaOH}$ concentrations below $0.01 \mathrm{~N}$ led to large amounts of bisazo dyestuff, which precipitated from the solution due to its low solubility.

The results in the Table also show that there is a tendency towards obtaining a smaller proportion of ortho-monoazo derivative at higher $\mathrm{NaOH}$ concentrations. The ratio ortho/para derivatives in the product seems to be only slightly affected by mixing. Both ortho and para have similar extinction coefficients in the VIS region. The main difference in their absorption spectra is found in the UV at wavelengths where 1-naphthol, pyridine, and impurities coming probably from the thermal decomposition of the diazonium salt also absorb. Therefore, it was difficult in some cases to determine the amount of each component (ortho and para) separately.

At speeds above $5000 \mathrm{rpm}$ (energy dissipation rate $>49 \mathrm{~W} / \mathrm{kg}$ ), the curves $X$ vs. $N$ tend to level off (Fig. 3), i.e. the chemically controlled regime is probably reached. Since the ratio paralortho $\gg 1$, then $k_{1, p} \gg k_{1,0}$, and, therefore, the formation of the bisazo compound should go mainly via Eqn. 7. Using a stopped-flow apparatus with optical detection, it was possible to measure $k_{1}=k_{1,0}+k_{1,0}$. The values obtained were $2.7 \times 10^{3}$ and $2.2 \times 10^{3}$ $\mathrm{mol}^{-1} \cdot \mathrm{m}^{3} \cdot \mathrm{s}^{-1}$, measured in 0.025 and $0.0125 \mathrm{~N} \mathrm{NaOH}$, respectively, using an excess of 1-naphthol (pseudo first-order con-

Table. Product Distribution ((moles of $i /$ total moles) $\times 100$, values $\pm 0.5 \%)$ for the Azo-Coupling of $1-$ Naphthol (1) and Diazotized Aniline

\begin{tabular}{|c|c|c|c|c|c|}
\hline \multirow[t]{2}{*}{$\mathrm{NaOH}$ concentration } & \multirow[t]{2}{*}{$N[\mathrm{rpm}]$} & \multicolumn{3}{|c|}{ Monoazo [\%] } & \multirow[t]{2}{*}{ Bisazo $[\%]$} \\
\hline & & para & ortho & total & \\
\hline $0.025 \mathrm{~N}$ & $\begin{array}{l}1000 \\
2000 \\
3000 \\
4000 \\
6000 \\
8000\end{array}$ & $\begin{array}{l}84.8 \\
86.2 \\
- \\
- \\
88.0 \\
90.0\end{array}$ & $\begin{array}{l}9.7 \\
9.1 \\
- \\
- \\
8.7 \\
7.5\end{array}$ & $\begin{array}{l}94.5 \\
95.3 \\
96.1 \\
96.1 \\
96.7 \\
97.5\end{array}$ & $\begin{array}{l}5.5 \\
4.7 \\
3.9 \\
3.9 \\
3.3 \\
2.5\end{array}$ \\
\hline $0.05 \mathrm{~N}$ & $\begin{array}{r}700 \\
1000 \\
5000 \\
8000\end{array}$ & $\begin{array}{l}91.5 \\
94.0 \\
94.8 \\
95.6\end{array}$ & $\begin{array}{l}5.7 \\
4.5 \\
4.3 \\
3.9\end{array}$ & $\begin{array}{l}97.2 \\
98.5 \\
99.1 \\
99.5\end{array}$ & $\begin{array}{l}2.8 \\
1.5 \\
0.9 \\
0.5\end{array}$ \\
\hline $0.10 \mathrm{~N}$ & $\begin{array}{l}1000 \\
2000 \\
4000 \\
5000 \\
6000\end{array}$ & $\begin{array}{l}- \\
94.5 \\
95.2 \\
96.5 \\
-\end{array}$ & $\begin{array}{l}- \\
5.4 \\
4.6 \\
3.1 \\
-\end{array}$ & $\begin{array}{l}99.0 \\
99.9 \\
99.8 \\
99.6 \\
99.5\end{array}$ & $\begin{array}{l}1.0 \\
0.1 \\
0.2 \\
0.4 \\
0.5\end{array}$ \\
\hline
\end{tabular}




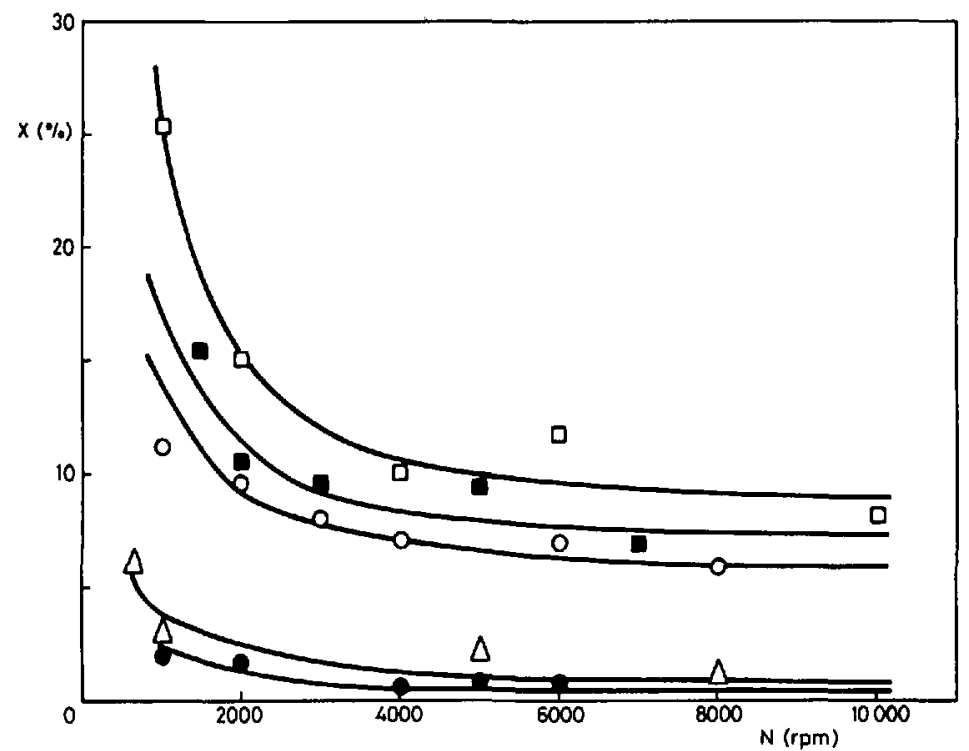

Fig. 3. Fraction of diazotized aniline present in the product as bisazo derivative as a function of the mixer speed at different $\mathrm{NaOH}$ concentrations. $\bullet: 0.1 \mathrm{~N}, \triangle: 0.05 \mathrm{~N}, 0: 0.025 \mathrm{~N}, \square 0.02 \mathrm{~N}$, 11: $0.01 \mathrm{~N}$.

ditions) at $25^{\circ}$. Kropacova et al. [7] report a value of $0.6 \times 10^{3} \mathrm{~mol}^{-1} \cdot \mathrm{m}^{3} \cdot \mathrm{s}^{-1}$ at $20^{\circ}$ and $0.05 \mathrm{M}$ ionic strenght. This value, however, does not include the base catalysis effect which appears to be the cause of the higher values of $k_{1}$ obtained from our measurements. Attempts were made to measure $k_{2,0}$. Nevertheless the formation of bisazo dyestuff seems to be more complicated than suggested by Eqn. 7. In $0.025 \mathrm{~N}$ $\mathrm{NaOH}$, two reaction steps were detected: one in the $0.1-1 \mathrm{~s}$ range and a much slower one in the $1-10 \mathrm{~s}$ range at reactant concentrations of $0.5 \mathrm{~mol} \cdot \mathrm{m}^{-3}$.

Mixing effects on chemical reactions are expected when the half lifetimes of reaction $\left(t_{\mathrm{R}}\right)$ and diffusion $\left(t_{\mathrm{D}}\right)$ are comparable (fast or diffusion-reaction regime), or when $t_{\mathrm{D}} \gg t_{\mathrm{R}}$ (instantaneous or diffusion-controlled regime). Theoretical models predict half lifetimes for diffusion, which are given by:

$t_{\mathrm{D}} \simeq 2(v / e)^{1 / 2} \operatorname{arcsinh}(0.05 \mathrm{Sc})$

where $S c=$ Schmidt number $=v / D ; v$ is the kinematic viscosity, $D$ is the molecular diffusivity, and $e$ is the energy dissipation rate [15].

At a speed of, for example, $1000 \mathrm{rpm}$, $e=0.4 \mathrm{~W} / \mathrm{kg}$. For aqueous solutions at room temperature [15], $v=10^{-6} \mathrm{~m}^{2} \cdot \mathrm{s}^{-1}$, $D=7.8 \times 10^{-10} \mathrm{~m}^{2} \cdot \mathrm{s}^{-1}, S c=1280$, we obtain $t \simeq 16 \mathrm{~ms}$.
When the diazotized aniline solution enters the reaction zone (before blending with the bulk), it has a concentration of 13 $\mathrm{mol} \cdot \mathrm{m}^{-3}$ and an acidic $\mathrm{pH}$. The initial concentration of 1 -naphthol is $1.39 \mathrm{~mol} \cdot \mathrm{m}^{-3}$. At these concentration levels, the primary reaction presents half lifetimes between 0.03 and $0.3 \mathrm{~ms}$. At the same concentrations, the half lifetime for the secondary reaction (only faster step considered) may well be in the range 4-400 ns.

Based on the half lifetimes for the reactions (primary and secondary) and diffusion, one may expect that poor mixing:

a) slows down the formation rate of monoazo dyestuff, $t_{\mathrm{D}} \gg t_{\mathrm{R}}$, and

b) makes the reaction monoazo $\rightarrow$ bisazo occur in the diffusion-reaction regime, $t_{\mathrm{D}} \simeq t_{\mathrm{R}}$, with the consequent alteration of the product distribution relative to the chemical or slow reaction regime.

Another factor directly related to mixing, which can influence the product distribution, is the existence of local $\mathrm{pH}$ gradients. Indeed, the azo-coupling reaction liberates protons which under certain circumstances may produce local $\mathrm{pH}$ values, which are lower than those in the bulk solution [16]. Thus, in the azo-coupling reaction of 1-naphthol and diazotised aniline, poor mixing conditions would favour the formation of ortho-monoazo and bisazo dyestuffs.

\section{Conclusions}

The product distribution of fast consecutive, competitive reactions may be influenced by mixing. An assessment of the mixing conditions, under which the reaction takes place, is convenient. The evaluation of the half lifetimes for diffusion and reaction gives an idea of the conditions, under which a mixing effect may be expected.

The authors wish to thank Prof. J.R. Bourne for useful discussion of the manuscript.
Received: April 9, 1990

[1] See for example: a) E. L. Paul, R. E. Treybal, $A$ /. ChE.J. 1971, 17, 718; b) S. B. Hanna, E. Hunziker, T. Saito, H. Zollinger, Helv. Chim. Acta 1969, 52,1537; c) A. Zoulalian, J. Villermaux, $A d$. Chem. 1974, 133, 348; d) J.R. Bourne, F. Kozicki, P. Rys, Chem. Eng. Sci. 1981, 36, 1643.

[2] H. Zollinger, 'Azo and Diazo Chemistry', Interscience, New York, 1961.

[3] H. E. Fiertz, H. Bruetsch, Helu. Chim. Acta 1921, 4,375 .

[4] L.N. Ogoleva, B.I, Stepanov, Zh. Organ. Khim. 1961, $I, 2083$.

[5] Y. Bansho, L. Saito, T. Yagi, Kogyo Kagaku Zasshi 1960, 63, 1597 .

[6] S. Kishimoto, T. Hirashima, O. Manabe, H. Hiyama, Kogyo Kugaku Zasshi 1968, 71, 1195.

[7] H. Kropacova, J. Panchartek, V. Sterba, K. Valter, Collect. Caech. Chem. Commun. 1970, 35, 3287.

[8] M. L. Crosslcy, R.H. Kienle, C.H. Benbrook, $\boldsymbol{J}$. Am. Chem. Soc. 1940, 62, 1400.

[9] D. Schulte-Frohlinde, H. Blume, 7. Phys. Chem. Neue Folge 1968, 59, 299.

[10] Th. Zincke, H. Bindewald, Ber. Deutsch. Chem. Ges. 1884, $17,3026$.

[11] O.N. Witt, J. Dedichen, Ber. Dewesch. Chem. Ges. 1897, 30, 2657.

[12] E. Bamberger, F. Heimberg, Ber, Deutsch. Chem. Ges. 1895, $28,1894$.

[13] J.R. Bourne, J. Garcia-Rosas, Chem. Eng. Res. Des. 1986, 64, 11 .

[14] O. Levenspiel, 'Chemical Reaction Engincering', 2nd edn., Wiley \& Sons, New York, 1972.

[15] J. R. Bourne, K. Ravindranath, S. Thoma, J. Org. Chem. 1988, 53, 5166.

[16] H. Belevi, J.R. Bourne, P. Rys, Helv. Chim. Actu $1981,64,1599$. 